\title{
Article \\ QED Response of the Vacuum
}

\author{
Gerd Leuchs $^{1,2, *}$ (D), Margaret Hawton ${ }^{3}$ (D), and Luis L. Sánchez-Soto ${ }^{1,4}$ (D) \\ 1 Max-Planck-Institut für die Physik des Lichts, Staudtstraße 2, 91058 Erlangen, Germany \\ 2 Institute for Applied Physics, Russian Academy of Sciences, 630950 Nizhny Novgorod, Russia \\ 3 Department of Physics, Lakehead University, Thunder Bay, ON P7B 5E1 Ontario, Canada; \\ mhawton@lakeheadu.ca \\ 4 Departamento de Óptica, Facultad de Física; Universidad Complutense, 28040 Madrid, Spain; \\ lsanchez@ fis.ucm.es \\ * Correspondence: gerd.leuchs@mpl.mpg.de
}

\begin{abstract}
We present a new perspective of the link between QED and Maxwell's equations. We demonstrate that the interpretation of $\mathbf{D}=\varepsilon_{0} \mathbf{E}$ as vacuum polarization is consistent with QED. A free electromagnetic field polarizes the vacuum, but the polarization and magnetization currents cancel giving zero source current. The speed of light is a universal constant, while the fine structure constant, which couples the electromagnetic field to matter, runs as it should.
\end{abstract}

Keywords: Quantum vacuum; Linear response; QED

\section{Introduction}

In quantum electrodynamics (QED) the vacuum is a dynamical entity, in the sense that there is a rich variety of virtual processes that can take place in it [1-3]. There are several observable effects that manifest themselves when the vacuum is perturbed in specific ways: vacuum fluctuations lead to shifts in the energy level of atoms (Lamb shift) [4], changes in the boundary conditions produce particles (dynamical Casimir effect) [5], and accelerated motion and gravitation can create thermal radiation (Unruh [6] and Hawking [7] effects).

QED conceives vacuum fluctuations as particle-antiparticle pairs that appear spontaneously, violating the conservation of energy according to the Heisenberg uncertainty principle. These pairs play a role in the value of the permittivity of the vacuum $\varepsilon_{0}$ : a photon will feel the presence of those pairs much the same as in a dielectric. This idea can be traced back to the time-honored works of Furry and Oppenheimer [8], Weisskopf and Pauli $[9,10]$ and Dicke [11], who contemplated the prospect of treating the vacuum as a medium with electric and magnetic polarizability.

This approach has been recently adopted [12-14] to calculate ab initio $\varepsilon_{0}{ }^{1}$ by using the same technique employed to determine the permitivitty in a dielectric. This has prompted significant research interest in adopting an oscillator model for the virtual pairs in order to assess some properties of the vacuum [15-22] . Interestingly, the possibility that a charged pair can form an atomic bound state (which can thus be well approximated by an oscillator) was discussed by Ruark [23] and further elaborated by Wheeler [24].

In the standard relation $\mathbf{D}=\varepsilon_{0} \mathbf{E}+\mathbf{P}$, the first term on the right-hand side is often referred to as the polarization of the bare vacuum. In QED, however, the polarization of the virtual pairs is added as part of $\mathbf{P}$. Because the new term is dispersionless like $\varepsilon_{0} \mathbf{E}$, the electric field can be rescaled to formally recover the initial equation. We suggest that the polarization of the virtual pairs should instead be identified with the first term $\varepsilon_{0} \mathbf{E}$. This is a paradigm shift in our physical picture of the vacuum. One consequence of this procedure is that in the

In Gaussian units the permittivity of the vacuum is inconspicuously "1". But whatever the unit system, this permittivity calls for a physical interpretation. 


\section{Vacuum polarization in QED}

As heralded in the Introduction, the vacuum in QED acts like a dielectric medium where the virtual pairs shield the original point charges. In this Section, the vacuum contribution to the dielectric permittivity $\varepsilon_{0}$ and the magnetic permeability $\mu_{0}$ will be calculated by incorporating them into the electromagnetic Lagrangian. In QED the bare potentials $A_{0}^{\mu}$ and charge $e_{0}$ are rescaled to give the physical four-potential $A^{\mu}$ and the physical electron charge $e$; that is,

$$
A_{0}^{\mu} \equiv \sqrt{Z_{3}} A^{\mu}, \quad e_{0} \equiv \sqrt{Z_{3}} e .
$$

This rescaling is at the basis of the renormalization program. The renormalized QED Lagrangian density will be written as

$$
\mathscr{L}_{\mathrm{QED}}=\mathscr{L}_{\text {Maxwell }}+\mathscr{L}_{\text {Dirac }}+\mathscr{L}_{\text {int }} .
$$

Details of $\mathscr{L}_{\text {Dirac }}$ are omitted because renormalization of the masses will not be discussed here. The interaction term is

$$
\mathscr{L}_{\text {int }}=-j^{\mu} A_{\mu} .
$$

Here, $j^{\mu}$ is the current due to real charges, while the current density $j_{\mathrm{v}}^{\mu}$ due to the creation of virtual pairs and the $Z_{3}-1$ counterterm are incorporated into $\mathscr{L}_{\text {Maxwell }}$. Together, they describe the reduction in vacuum polarization relative to its maximum value at $k^{2}=0$. The current induced in the vacuum by the four-potential $A_{v}$ due to virtual pairs of type $\mathfrak{s}$ (where $\mathfrak{s}$ corresponds to the possible different leptons) is

$$
j_{v, \mathfrak{s}}^{\mu}(k)=c^{2} \varepsilon_{0} \Pi_{\mathfrak{s}}\left(k^{2}\right)\left(k^{2} g^{\mu v}-k^{\mu} k^{v}\right) A_{v}(k) .
$$

where $g^{\mu v}$ is the metric tensor, with diagonal $(1,-1,-1,-1)$, and $\Pi_{\mathfrak{s}}\left(k^{2}\right)$ is the QED vacuum "polarization" of a s-type particle. If $A_{v}(k)$ describes real photons, the on-shell condition $k^{2}=0$ is satisfied. This is a generalization of the usual textbook treatment of electron-position virtual pairs [26,27], extended to other fermions.

The Feynman diagram in Fig. 1 is a pictorial representation of vacuum polarization in the one-loop approximation. The wavy lines represent an electromagnetic field, while a vertex represents the interaction of the field with the fermions, which are represented by the internal lines. The loop labelled 1 represents a virtual electron-positron pair created at space-time point $x_{1}=\left(c t_{1}, \mathbf{x}_{1}\right)$ and annihilated at $x_{2}=\left(c t_{2}, \mathbf{x}_{2}\right)$. The loop labelled 2 represents creation of a muon-antimuon pair and so on. The sum over single virtual pairs in the linear approximation, where $\Pi$ is $A$-independent, is

$$
\Pi\left(k^{2}\right)=\sum_{\mathfrak{s}}^{\text {e.p. }} \Pi_{\mathfrak{s}}\left(k^{2}\right) .
$$




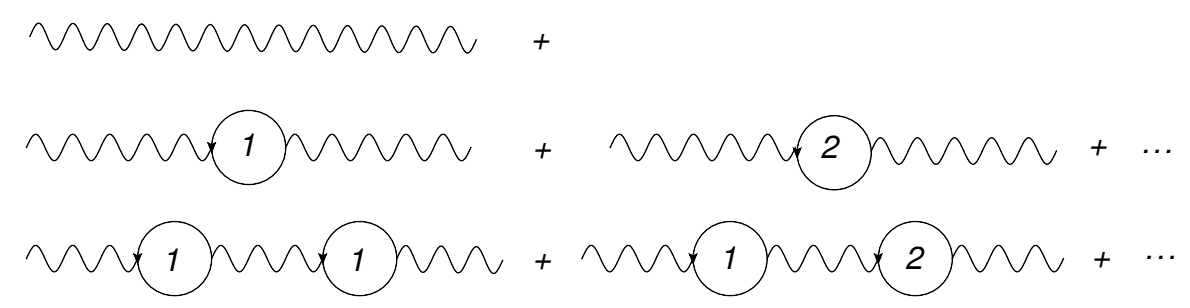

Figure 1. Vacuum polarization in the one-loop approximation. All types of virtual pairs in Nature (labeled as $1,2, \ldots)$ polarize the vacuum. This polarization is maximal for a free electromagnetic field for which $\omega=|\mathbf{k}| c$.

In the on-shell counterterm

$$
Z_{3}-1=\Pi(0)
$$

The vacuum polarization $\Pi_{\mathfrak{s}}\left(k^{2}\right)$ is a divergent sum over fermion momenta and naive introduction of a cut-off leads to a physically unreasonable result (the photon mass is infinite) [26,28]. Since observations are made near $k^{2}=0$, the vacuum polarization relative to its on-shell value,

$$
\widehat{\Pi}\left(k^{2}\right) \equiv \Pi\left(k^{2}\right)-\Pi(0)
$$

is calculated instead. Writing $Z_{3}$ as $1+\left(Z_{3}-1\right)$ to separate it into fully polarized and polarization-reduction terms and integrating $j_{\mathrm{v}}^{\mu} A_{\mu}$ by parts to get its $E^{2}-c^{2} B^{2}$ form [27], the Maxwell Lagrangian density becomes

$$
\mathscr{L}_{\text {Maxwell }}=\frac{1}{2} \varepsilon_{0}\left(k^{2}\right)\left(\mathbf{E}^{2}-c^{2} \mathbf{B}^{2}\right)
$$

where

$$
\varepsilon_{0}\left(k^{2}\right)=\varepsilon_{0}\left[1+\widehat{\Pi}\left(k^{2}\right)\right]
$$

47 and we have used $\mu_{0}^{-1}=c^{2} \varepsilon_{0}$.

To second order in perturbation theory, the vacuum polarization relative to it maximum values at $k^{2}=0$ can be approximated by

$$
\widehat{\Pi}_{2}\left(k^{2}\right)=-\frac{6}{12 \pi^{2} \hbar c} \sum_{\mathfrak{s}} q_{\mathfrak{s}}^{2} \int_{0}^{1} d x x(1-x) \ln \left[1-\frac{\hbar^{2} k^{2}}{m_{\mathfrak{s}}^{2} c^{2}} x(1-x)\right] \simeq-\frac{1}{12 \pi^{2} \hbar c} \sum_{\mathfrak{s}} q_{\mathfrak{s}}^{2} \ln \left(\frac{\hbar^{2} k^{2}}{A m_{\mathfrak{s}}^{2} c^{2}}\right)
$$

where the rightmost equation is valid when $\hbar^{2}\left|k^{2}\right| \gg m_{\mathfrak{s}}^{2} c^{2}$.

In classical electromagnetism $\mathbf{D}=\varepsilon_{0} \mathbf{E}$. This relationship is maintained here except that, with running, $\varepsilon_{0}\left(k^{2}\right) \leq \varepsilon_{0}$. The linear response of the vacuum is then described in reciprocal $k$ space by

$$
\mathbf{D}(k)=\varepsilon_{0}\left(k^{2}\right) \mathbf{E}(k), \quad \mathbf{H}(k)=c^{2} \varepsilon_{0}\left(k^{2}\right) \mathbf{B}(k) .
$$

The Maxwell equations in vacuum can be derived from the Lagrangian (2), following the standard method. They take the usual form in $k$-space:

$$
i \mathbf{k} \cdot \mathbf{D}(k)=\rho(k), \quad i \mathbf{k} \times \mathbf{H}(k)+i \frac{\omega}{c} \mathbf{D}(k)=\mathbf{j}(k) .
$$
written as

The photon two-point correlation function is found by solving (4) for $A_{v}$ [27]. Using Eq. (9), it can be

$$
i D_{F}^{\mu v}(k)=\frac{-i g^{\mu \nu}}{\varepsilon_{0}\left(k^{2}\right)\left(k^{2}+i \eta\right)} .
$$

This $D_{F}$ is the response to a $\delta$-function source in real space and hence to a constant driving force in $k$-space. Equation (13) is then a Green function satisfying Maxwell's wave equation. In the Lorenz gauge, and in $k$-space this is

$$
c^{2} \varepsilon_{0}\left(k^{2}\right) k^{2} A^{\mu}(k)=-j^{\mu}
$$


where $j^{\mu}$ is $k$ independent. The matter-field coupling constant is $\alpha=e^{2} /\left(4 \pi \hbar c \varepsilon_{0}\right)$. Here, we hold $e$ constant and incorporate running into $\varepsilon_{0}\left(k^{2}\right)$, so that

$$
\alpha\left(k^{2}\right)=\frac{e^{2}}{4 \pi \hbar \varepsilon_{0}\left(k^{2}\right)} .
$$

Since $\varepsilon_{0}\left(k^{2}\right)^{-1}$ contains all powers of $e^{2}$, it incorporates summation over all numbers of pairs as sketched in Fig. 1 and used in the calculation of (13). When restricted to an energy scale $\mathscr{E}_{\max }$, the sum is over all fermions of mass less than $\mathscr{E}_{\max } / c^{2}$ [29-31]. Running of $e^{2} / \varepsilon_{0}\left(k^{2}\right)$ is in most ways equivalent to running of the square of effective charge in conventional QED, but the physical interpretation is different. In a dielectric it is possible to have $\varepsilon_{0}<0$, but $e_{\text {eff }}^{2}<0$ makes no physical sense.

The dielectric properties of vacuum differ from those of a material medium in two important ways: $\ln \left(k^{2}\right)$ dependence replaces the usual $\omega$ dependence and Lorentz invariance requires that $\varepsilon_{0}\left(k^{2}\right) \mu_{0}\left(k^{2}\right)=1 / c^{2}$. The speed $c$ is a universal constant ${ }^{2}$ whereas the coupling constant $\alpha\left(k^{2}\right)$ runs. On the photon mass shell $k^{2}=0$, so a free photon always sees $\varepsilon_{0}$ and there is no running. Both the polarization $\mathbf{D}$ and the magnetization $\mathbf{H}$ are nonzero; however, $\mathbf{j}_{\mathrm{v}}=0$ as it must for propagation in free space. A free electromagnetic wave polarizes and magnetizes the vacuum but the polarization current exactly cancels the magnetization current ${ }^{3}$.

\section{Connection with an oscillator model}

An individual loop in Fig. 1 is analogous to a single polarizable atom with center of mass momentum $\hbar k$. If, for simplicity, we set $k=0$, the computation of the Feynmann diagrams involve integrals of the form $\int d^{4} q\left[q^{2}+\left(\frac{m_{\mathfrak{s}} c}{\hbar}\right)\right]^{-2}$, which entail an exponential decay, $\exp \left[-\left(m_{\mathfrak{s}} c / \hbar\right)|\mathbf{x}|\right]$, in real space. Therefore, the "radius" of a virtual atom is of order $\hbar / m_{\mathfrak{s}} c$. All in all, this suggests that the virtual pairs can be modelled as oscillating dipoles with frequency $m c^{2} / \hbar$ and volume of order $\left(\hbar / m_{\mathfrak{s}} c\right)^{3}$.

When a virtual pair is created by a photon with frequency $\omega$ its excess energy is, according the Heisenberg uncertainty principle, $\Delta E \geq 2 m c^{2}-\hbar \omega$, so it can exist only for a time $t_{2}-t_{1} \leq \hbar / \Delta E$. ${ }^{4}$ Similar restrictions apply to the distance that the virtual particles can travel.

Based on the uncertainty principle and this simple oscillator model, one can readily find that the permittivity of vacuum can be expressed as $[12,13]$

$$
\varepsilon_{0}=f \frac{1}{\hbar c} \sum_{\mathfrak{s}}^{\text {e.p. }} q_{\mathfrak{s}}^{2},
$$

where $f$ is a geometrical factor of order unity, $q_{\mathfrak{s}}$ is charge and the sum is over all elementary charged particles.

This is consistent with QED, as discussed in the previous Section. Indeed, at large $k^{2}$ and to second order in perturbation theory as in Eq. (10), we have that

$$
\varepsilon_{0}(k) \simeq \varepsilon_{0}-\frac{1}{12 \pi^{2} \hbar c} \sum_{\mathfrak{s}}^{\text {e.p. }} q_{\mathfrak{s}}^{2} \ln \left(\frac{\hbar^{2} k^{2}}{m_{\mathfrak{s}}^{2} c^{2}}\right) .
$$

We know that at high momentum (or energy) scale, the coupling constant $\alpha(k)$ in QED becomes infinity [34,35]. If $\Lambda$ is the value of that momentum (which is usually called the Landau pole [36]), then

$$
\varepsilon_{0}=\frac{1}{12 \pi^{2} \hbar c} \sum_{\mathfrak{s}}^{\text {e.p. }} q_{\mathfrak{s}}^{2} \ln \left(\frac{\hbar^{2} \Lambda^{2}}{m_{\mathfrak{s}}^{2} c^{2}}\right),
$$

2 Interestingly, even without imposing this requirement and when using the oscillator model in next section to independently calculate $\mu_{0}$, the resulting speed of light is independent of how many types of elementary particles contribute and agrees with the limiting speed in Lorentz's equations [13].

3 This bears some resemblance with the interference between the emitted electric and magnetic dipole waves leading to forward scattering only and no back scattering, the property of Huygens waves which typically appear in materials with comparably strong electric and magnetic interaction, which holds for the vacuum [32] and was recently rediscovered for metamaterials [33].

4 While a real pair cannot be created by absorbing a photon due to simultaneous conservation of energy and momentum, this restriction does not apply to the ephemeral creation of virtual pairs. 
and hence the fudge factor $f$ in the oscillator model is given by

$$
f=\frac{1}{12 \pi^{2}} \frac{\sum_{\mathfrak{s}}^{\text {e. p. }} q_{\mathfrak{s}}^{2} \ln \left(\frac{\hbar^{2} \Lambda^{2}}{m_{\mathfrak{s}}^{2} c^{2}}\right)}{\sum_{\mathfrak{s}}^{\text {e. p. }} q_{\mathfrak{s}}^{2}} .
$$

For the standard model $\sum q_{\mathfrak{s}}^{2} / e^{2}=9$ and with two additional charged Higgs particles of $m c^{2} \simeq 5 \times 10^{11} \mathrm{eV}, f \simeq 1$ and all of $\varepsilon_{0}$ is vacuum polarization if $\log (\hbar \Lambda / c)=35$.

If running is neglected, the on-shell Maxwell equations in a medium with charges and current sources are

$$
\begin{array}{ll}
\nabla \cdot \mathbf{B}(\mathbf{r}, t)=0, & \nabla \times \mathbf{E}(\mathbf{r}, t)+\partial \mathbf{B}(\mathbf{r}, t) / \partial t=0, \\
\nabla \cdot \mathbf{D}(\mathbf{r}, t)=\rho(\mathbf{r}, t), & \nabla \times \mathbf{H}(\mathbf{r}, t)-\partial \mathbf{D}(\mathbf{r}, t) / \partial t=\mathbf{j}(\mathbf{r}, t) .
\end{array}
$$

as in classical electromagnetic theory. Equations (20) allow us to define the four-vector potential that drives the creation of the pairs. Equations (21) simply say that charge density is the divergence of polarization and the current is the sum of its polarization and magnetization parts.

Zeldovich considered a Lagrangian in which all of the electromagnetic term comes from the interaction of the particle with the field and came to an interesting conclusion [37]: In the absence of vacuum polarization electric and magnetic fields act on Dirac fermions, but there is no field energy and no electromagnetic wave propagation. In an absolute void it makes no sense to talk about Maxwell's equations or light propagation. Only after vacuum polarization is introduced does the effective Lagrangian give the ME, EM waves travelling at the speed of light and photons.

\section{Gottfried-Weisskopf dielectric model}

Gottfried and Weisskopf [38] introduced a toy model to understand the physical mechanisms involved in vacuum polarization. In this simple picture, one assumes that the bare charge $e_{0}$ is uniformly distributed in a small sphere of radius $a\left[a \ll \hbar /\left(m_{e} c\right]\right.$. When this charge distribution is surrounded by a spherical shell of inner radius $r>a$, filled with a dielectric medium permittivity $\varepsilon$, an induced charge of opposite sign appears on the inner surface of the shell at radius $r$, canceling part of the charge $e_{0}$ for an observer at a distance larger than $r$. Obviously, at the outer surface of the dielectric medium, an equal charge of opposite sign appears. However, if the charge is measured from within the medium it will appear to be reduced, precisely by the permittivity $\varepsilon$ [39].

When one considers the vacuum, one has to take into account that it extends to infinity. Now, the permittivity depends on the distance $r$ to the charge. This is so because at $r$ only those virtual pairs having Compton wavelengths $\lambda \lesssim r$ contribute. We can interpret his model from a QED viewpoint. To this end, we define the relationship between $\mathbf{D}$ and $\mathbf{E}$ at charge separations $r \gg \hbar /\left(m_{e} c\right)$ where the coupling strength can be measured; that is, $\varepsilon_{0}\left(r \gg \hbar /\left(m_{e} c\right)\right) \equiv \varepsilon_{0}$ at large distances or, equivalently, small momenta, where the vacuum is maximally polarized. With polarization included the dielectric permittivity is $\varepsilon_{0}$ at the physical scale. In consequence, $\widehat{\Pi}$ is the reduction in polarization.

Since $\varepsilon_{0}\left(k^{2}\right)$ is not a constant, the exact relationship between $\mathbf{D}$ and $\mathbf{E}$ is nonlocal in $r$-space. It is not, in general, correct to write the potential as $\Phi=e / 4 \pi \varepsilon_{0}(r) r$. The simplest example in which running coupling can be expressed as an explicit local function of $r$ is a static charge, say $+e$. In the Coulomb gauge $\mathbf{E}\left(\mathbf{k}^{2}\right)=-\mathbf{k} \Phi\left(\mathbf{k}^{2}\right)$ and $\omega=0$. Equation (12), with $k^{2}=-\mathbf{k}^{2}$, then gives

$$
\Phi\left(\mathbf{k}^{2}\right)=\frac{e}{\mathbf{k}^{2} \varepsilon_{0}\left(\mathbf{k}^{2}\right)},
$$

which in $\mathbf{r}$-space reads

$$
\Phi(r)=\int \frac{d^{3} \mathbf{k}}{(2 \pi)^{3}} \frac{e}{\mathbf{k}^{2} \varepsilon_{0}\left(\mathbf{k}^{2}\right)} \exp (i \mathbf{k} \cdot \mathbf{r}),
$$


equivalent to Eq. (7.93) in [27].

For electron-positron pairs alone the magnitude of $\widehat{\Pi}$ is very much less that 1 so that $\varepsilon_{0}^{-1}\left(k^{2}\right)$ is approximately $\left[1+\widehat{\Pi}\left(k^{2}\right)\right] / \varepsilon_{0}$ and $[40]$

$$
\Phi(r) \simeq \frac{e}{4 \pi \varepsilon_{0} r} \times \begin{cases}1+\frac{2 \alpha}{3 \pi} \ln \left(\frac{\hbar}{m_{e} c r}\right)-\gamma-\frac{5}{6} & r \ll \hbar /\left(m_{e} c\right), \\ 1+\frac{\alpha}{4 \sqrt{\pi}} \frac{e^{-2 m_{e} c r} / \hbar}{\left(m_{e} c r / \hbar\right)^{3 / 2}} & r \gg \hbar /\left(m_{e} c\right),\end{cases}
$$

where $m_{e}$ is the mass of the electron, $r$ is the distance from the fixed charge and $\gamma=0.577$ is Euler's constant. The dielectric constant $\varepsilon_{0}$ decreases with increasing $\mathbf{k}^{2}$ or decreasing $r$. For $r<\hbar /\left(m_{e} c\right)$ the Coulomb interaction becomes stronger as the charges approach each other.

\section{Conclusion}

We have verified that QED vacuum is a polarizable medium with dielectric constant $\varepsilon_{0}\left(k^{2}\right) \leq \varepsilon_{0}$. Lorentz invariance requires $\mu_{0}\left(k^{2}\right) \varepsilon_{0}\left(k^{2}\right)=1 / c^{2}$, where the speed of light is a universal constant, which is also implied by the dielectric/diamagnetic model of the vacuum [13]. For a free photon $k^{2}=0$ so there is no change in $\varepsilon_{0}\left(k^{2}\right)$ with photon energy; it does not run. Since $E=B / c \neq 0$ for any free EM wave it will polarize and magnetize the vacuum but their contributions to the electric current cancel.

This dielectric model predicts no new observable results but it suggests a paradigm shift in our physical picture of the vacuum. Any electromagnetic field creates virtual pairs of all charged elementary particle types in Nature. At the scale of classical electromagnetism and quantum optics $\varepsilon_{0}=\varepsilon_{0}(0)$ and the vacuum is maximally polarized.

GL acknowledges financial support by the Ministry of Education and Science of the Russian Federation megagrant No. 14.W03.31.0032. LLSS acknowledges financial support from Spanish MINECO (Grants FIS2015-67963-P and PGC2018-099183-B-I00).

1. Borchers, H.J.; Haag, R.; Schroer, B. The vacuum state in quantum field theory. Il Nuovo Cimento 1963, $29,148-162$. doi:10.1007/BF02750157.

2. Sciama, D.W., The Philosophy of Vacuum; Clarendon: Oxford, 1991; chapter The Physical Significance of the Vacuum State of a Quantum Field, pp. 137-158.

3. Milonni, P.W., The Quantum Vacuum; Academic Press: San Diego, 1994.

4. Lamb, W.E.; Retherford, R.C. Fine Structure of the Hydrogen Atom by a Microwave Method. Phys. Rev. 1947, 72, 241-243. doi:10.1103/PhysRev.72.241.

5. Moore, G.T. Quantum Theory of the Electromagnetic Field in a Variable-Length One-Dimensional Cavity. J. Math. Phys. 1970, 11, 2679-2691. doi:10.1063/1.1665432.

6. Unruh, W.G. Notes on black-hole evaporation. Phys. Rev. D 1976, 14, 870-892. doi:10.1103/PhysRevD.14.870.

7. Hawking, S.W. Particle creation by black holes. Commun. Math. Phys. 1975, 43, 199-220. doi:10.1007/BF02345020.

8. Furry, W.H.; Oppenheimer, J.R. On the Theory of the Electron and Positive. Phys. Rev. 1934, 45, $245-262$. doi:10.1103/PhysRev.45.245.

9. Pauli, W.; Weisskopf, V. Über die Quantisierung der skalaren relativistischen Wellengleichung. Helv. Phys. Acta 1934, 7, 709-731.

10. Weisskopf, V.F. Über die Elektrodynamik des Vakuums auf Grund des Quanten-Theorie des Elektrons. Kgl. Danske Videnskab Selskab Mat.-Fys. Medd. 1936, 14, 1-39.

11. Dicke, R.H. Gravitation without a Principle of Equivalence. Rev. Mod. Phys. 1957, 29, 363-376. doi:10.1103/RevModPhys.29.363.

12. Leuchs, G.; Villar, A.S.; Sánchez-Soto, L.L. The quantum vacuum at the foundations of classical electrodynamics. Appl. Phys. B 2010, 100, 9-13. doi:10.1007/s00340-010-4069-8. 
13. Leuchs, G.; Sánchez-Soto, L.L. A sum rule for charged elementary particles. Eur. Phys. J. D 2013, 67, 57. doi:10.1140/epjd/e2013-30577-8.

14. Urban, M.; Couchot, F.; Sarazin, X.; Djannati-Atai, A. The quantum vacuum as the origin of the speed of light. Eur. Phys. J. D 2013, 67, 58. doi:10.1140/epjd/e2013-30578-7.

15. AlMosallami, A. Time Contraction: The Possibility of Faster Than Light without Violation of Lorentz Transformation or Causality and the Vacuum Energy Dependent. Int. J. Modeern Theo. Phys. 2014, 3, 44-73.

16. Shivalingaswamy, T.; Rashmi, P. I am the speed of light c, you 'see' ....! EJPE 2017, 5, 51-58.

17. Mainland, G.B.; Mulligan, B. Theoretical calculation of the fine-structure constant and the permittivity of the vacuum. arXiv:1705.11068 2017.

18. Braun, D.; Schneiter, F.; Fischer, U.R. Intrinsic measurement errors for the speed of light in vacuum. Class. Quantum Gravity 2017, 34, 175009. doi:10.1088/1361-6382/aa8058.

19. Zhu, Y. Gravitational-magnetic-electric field interaction. Results Phys. 2018, 10, 794-798. doi:https://doi.org/10.1016/j.rinp.2018.07.029.

20. Razmi, H.; Baramzadeh, N.; Baramzadeh, H. Dispersive property of the quantum vacuum and the speed of light. Mod. Phys. Lett. A 2019, 34, 1950035. doi:10.1142/S0217732319500354.

21. Mainland, G.B.; Mulligan, B. How vacuum fluctuations determine the properties of the vacuum. J. Phys.: Conf. Series 2019, 1239, 012016. doi:10.1088/1742-6596/1239/1/012016.

22. Udem, T.; Maisenbacher, L.; Matveev, A.; Andreev, V.; Grinin, A.; Beyer, A.; Kolachevsky, N.; Pohl, R.; Yost, D.C.; Hänsch, T.W. Quantum Interference Line Shifts of Broad Dipole-Allowed Transitions. Ann. Phys. 2019, 531, 1900044. doi:10.1002/andp.201900044.

23. Ruark, A.E. Positronium. Phys. Rev. 1945, 68, 278-278. doi:10.1103/PhysRev.68.278.

24. Wheeler, J.A. Polyelectrons. Ann. N.Y. Acad. Sci. 1946, 48, 219-238. doi:10.1111/j.1749-6632.1946.tb31764.x.

25. Wilczek, F. The Lightness of Being; Basic Books: New York, 2008.

26. Itzykson, C.; Zuber, J.B. Quantum Field Theory; Dover: New York, 1980.

27. Peskin, M.E.; Schroeder, D.V. An Introduction to Quantum Field Theory; CRC Press: Boca Raton, 2018.

28. Bogoliubov, N.N.; Shirkov, D.V. The Theory of Quantized Fields; Interscience: New York, 1959.

29. Eidelman, S.; Jegerlehner, F. Hadronic contributions to (g-2) of the leptons and to the effective fine structure constant. Z. Phys. C 1995, 67, 585-601. doi:10.1007/BF01553984.

30. Hogan, C.J. Why the universe is just so? Rev. Mod. Phys. 2000, 72, 1149-1161. doi:10.1103/RevModPhys.72.1149.

31. Hoecker, A. The hadronic contribution to the muon anomalous magnetic moment and to the running electromagnetic fine structure constant at MZ -overview and latest results. Nucl. Phys. B Proc. Suppl. 2011, 218, 189-200. doi:https://doi.org/10.1016/j.nuclphysbps.2011.06.031.

32. Kerker, M.; Wang, D.S.; Giles, C.L. Electromagnetic scattering by magnetic spheres. J. Opt. Soc. Am. 1983, 73, 765-767. doi:10.1364/JOSA.73.000765.

33. Decker, M.; Staude, I. Resonant dielectric nanostructures: a low-loss platform for functional nanophotonics. $J$. Opt. 2016, 18, 103001. doi:10.1088/2040-8978/18/10/103001.

34. Gell-Mann, M.; Low, F.E. Quantum Electrodynamics at Small Distances. Phys. Rev. 1954, 95, $1300-1312$. doi:10.1103/PhysRev.95.1300.

35. Leuchs, G.; Hawton, M.; Sánchez-Soto, L.L. Quantum field theory and classical optics: determining the fine structure constant. J. Phys.: Conf. Ser. 2017, 793, 012017.

36. Landau, L.D.; Abrikosov, A.A.; Khalatnikov, I.M. On the removal of infinities in quantum electrodynamics. Dokl. Akad. Nauk SSSR 1954, 95, 497-502.

37. Zel'dovich, Y.B. Interpretation of Electrodynamics as a Consequence of Quantum Theory. JEPT Lett. 1967, $6,345-347$.

38. Gottfried, K.; Weisskopf, V.F. Concepts of Particle Physics; Vol. II, Oxford University Press, 1986.

39. Ashcroft, N.V.; Mermin, N.D. Solid State Physics; Hartcourt: Fort Worth, 1976.

40. Landau, L.D.; Lifshitz, E.M.; Pitaevskii, L.P. Relativistic Quantum Theory (part I); Vol. 4, Course of Theoretical Physics, Pergamon: London, 1973. 